\title{
The effects of antiviral treatment on breast cancer cell line
}

\author{
Madina Shaimerdenova ${ }^{1}$, Orynbassar Karapina ${ }^{2}$, Damel Mektepbayeva ${ }^{1}, K^{2}$ neth Alibek ${ }^{3}$ and Dana Akilbekova ${ }^{{ }^{*}}$
}

\begin{abstract}
Background: Recent studies have revealed the positive antiproliferative and cytotoxic effects of antiviral agents in cancer treatment. The real effect of adjuvant antiviral therapy is still controversial due to the lack of studies in biochemical mechanisms. Here, we studied the effect of the antiviral agent acyclovir on morphometric and migratory features of the MCF7 breast cancer cell line. Molecular levels of various proteins have also been examined.

Methods: To evaluate and assess the effect of antiviral treatment on morphometric, migratory and other cellular characteristics of MCF7 breast cancer cells, the following experiments were performed: (i) MTT assay to measure the viability of MCF7 cells; (ii) Colony formation ability by soft agar assay; (iii) Morphometric characterization by immunofluorescent analysis using confocal microscopy; (iv) wound healing and transwell membrane assays to evaluate migration and invasion capacity of the cells; (v) ELISA colorimetric assays to assess expression levels of caspase-3, E-cadherin and enzymatic activity of aldehyde dehydrogenase (ALDH).
\end{abstract}

Results: We demonstrate the suppressive effect of acyclovir on breast cancer cells. Acyclovir treatment decreases the growth and the proliferation rate of cells and correlates with the upregulated levels of apoptosis associated cytokine Caspase-3. Moreover, acyclovir inhibits colony formation ability and cell invasion capacity of the cancer cells while enhancing the expression of E-cadherin protein in MCF7 cells. Breast cancer cells are characterized by high ALDH activity and associated with upregulated proliferation and invasion. According to this study, acyclovir downregulates ALDH activity in MCF7 cells.

Conclusions: These results are encouraging and demonstrate the possibility of partial suppression of cancer cell proliferation using an antiviral agent. Acyclovir antiviral agents have a great potential as an adjuvant therapy in the cancer treatment. However, more research is necessary to identify relevant biochemical mechanisms by which acyclovir induces a potent anti-cancer effect.

Keywords: MCF7 breast cancer cell line, Acyclovir, Antiproliferative effect, ALDH activity

\section{Background}

Current cancer therapy includes the use of chemotherapeutic agents, surgery and radiation therapy. It is estimated that four types of viruses alone could cause $12 \%$ of cancer cases worldwide. These are human papillomavirus (HPV), hepatitis B (HBV), hepatitis C (HCV), and Epstein-Barr virus (EBV) [1]. Investigation of the virusassociated cancer serves as a unique platform for the development of novel strategies to prevent the development of infection that can predispose tumorigenesis. Studies on antiviral drug treatments demonstrate promising

\footnotetext{
* Correspondence: dana.akilbekova@nu.edu.kz

${ }^{1}$ National Laboratory Astana, Nazarbayev University, Qabanbay Batyr Avenue

53, Astana 010000, Kazakhstan

Full list of author information is available at the end of the article
}

results on the prognosis through the prevention of carcinogenesis. Administration of the antiviral agents in combination with the anticancer drugs is known for positively influencing the effectiveness of the treatment [2]. This combined therapy is termed as an adjuvant antiviral therapy [3]. Complex combinations of the chemotherapeutic agents together with the antiviral drugs are used to treat a number of infection-associated malignancies such as Kaposi sarcoma, hepatocellular carcinoma (HCC) and nasopharyngeal carcinoma [4, 5]. A study based on the electronic health records of 2671 adult participants diagnosed with chronic HBV infection from 1992 to 2011 also indicates that an antiviral treatment against chronic HBV 
infection markedly decreases the incidence of $\mathrm{HCC}$ in the treated patients [5].

Adjuvant antiviral therapy also has a reported antiproliferative effect in some types of cancer [2]. Treatment of breast cancer cells with ribavirin decreases the level of one of the biomarkers of this malignancy, eukaryotic translation initiation factor (eIF4E), which is usually elevated in more than $25 \%$ of cancer cases [6]. Ribavirin disrupts the structure of eIF4E, leading to the inhibition of cyclin D1 and expression of NBS1 oncogene [7, 8].

Namba et al. demonstrated the use of zidovudine, an antiviral drug, in combination with gemcitabine, a chemotherapeutic agent - in an attempt to overcome a gemcitabine resistance for the pancreatic cancer treatment. In this type of malignancy, the gemcitabine resistance is associated with a decreased level of human equilibrative nucleoside transporter 1 (hENT1) and acquisition of epithelial-to-mesenchymal transition (EMT) - like phenotype. The zidovudine adjunct therapy was shown to reverse both events in this study [9]. Furthermore, authors demonstrated that activation of Akt-GSK3 $\beta$-Snail mechanism, one of the major signaling pathways during gemcitabine resistance, is inhibited by zidovudine so that gemcitabineresistant cancer cells were resensitized.

Although there is a plethora of evidence suggesting the beneficial effect of the antiviral agents in cancer treatment, the therapeutic benefit of their use in cancer treatment remains a grey area due to the lack of studies of the biochemical mechanisms. Antiviral agents such as acyclovir and ribavirin have been reported to have a suppressive effect on the proliferation and ability to increase an apoptosis in various cancers [7, 8]. Acyclovir was discovered 40 years ago and remains one of the main existing therapies for herpes simplex virus (HSV) infections. This drug is a highly potent inhibitor of this virus and commonly used for the treatment of the infections caused by the herpes viruses, CMV and EBV. It also has a low toxicity for the normal cells [10].

In the present study, we propose to investigate how cancer cells respond to the antiviral agent as acyclovir in vitro and whether this treatment can affect the metastatic phenotype of cancer cells. We report results on the potential effect of acyclovir treatment on the cell proliferation, invasion capacity, cytotoxicity, and the expression of tumor suppressing genes.

\section{Methods}

\section{Cell culture}

Breast cancer cell line MCF7 (American Type Cell Collection, ATCC $\left.{ }^{\ominus} \mathrm{HTB}_{22^{\mathrm{TM}}}\right)$ and human breast epithelial primary cells (Celprogen, Benelux, Netherlands) were cultured in a complete media (CM) (Dulbecco's modified Eagle's medium (DMEM) (D6421, Sigma-Aldrich, St Louis, MO, USA) supplemented with $10 \%$ fetal bovine serum (12103C-500 ml, Sigma-Aldrich, Buchs, Switzerland), $100 \mathrm{U} / \mathrm{mL}$ penicillin, $100 \mu \mathrm{g} / \mathrm{mL}$ streptomycin and $25 \mathrm{ug} /$ $\mathrm{mL}$ Amphotericin B (SV30079.01, HyClone, Thermo Scientific, South Logan, Utah, USA) at $37{ }^{\circ} \mathrm{C}$ in $5 \% \mathrm{CO}_{2}$. Cells were subcultured every three days using $0.25 \%$ trypsin-EDTA (25-052-CI, Cellgro, Mediatech Inc, Manassas, VA, USA) for detachment.

\section{Treatment with Acyclovir}

Antiviral agent - acyclovir (ACV) in powder form was purchased from Sigma-Aldrich (PHR1254-1G, St Louis, MO, USA). A $10 \mathrm{mM}$ (stock) solution was prepared in phosphate buffered saline (PBS) and sterilized through filtering (0.45 $\mu \mathrm{M}$ PVDF $25 \mathrm{~mm}$ filters, (094.01.003, Isolab, Wertheim, Germany). Stock solution was stored at $-20{ }^{\circ} \mathrm{C}$. MCF7 cells were cultured in 12-well plate at 26,000 cells $/ \mathrm{cm}^{2}$ (92412, TPP, Switzerland) in the presence of $5 \mathrm{uM}$ acyclovir solution and incubated for $72 \mathrm{~h}$ at $37{ }^{\circ} \mathrm{C}$ in $5 \% \mathrm{CO}_{2}$. In a positive control experiment, cells were cultured in the absence of acyclovir. A control of acyclovir without cells was also conducted. All experiments were performed in triplicate.

\section{Viability assay}

Cell viability after the acyclovir treatment was evaluated with MTT (3-(4,5-dimethylthiazol-2- yl)-2,5-diphenyltetrazolium bromide (M5655-1G, Sigma Aldrich, St Louis, $\mathrm{MO}$, USA) assay. Concentration of $5 \mathrm{mg} / \mathrm{ml}$ was achieved by reconstituting MTT in DI water. After removal of the supernatant from the wells, $500 \mu \mathrm{l}$ of warm culture media and $50 \mu \mathrm{l}$ of MTT solution were added in each well for two hours at $37{ }^{\circ} \mathrm{C}$ in $5 \% \mathrm{CO}_{2}$. Then $400 \mu \mathrm{l}$ of media was removed and crystals of formazan were diluted with $500 \mu \mathrm{l}$ of dimethyl sulfoxide (DMSO) (D4540-100 ml, Sigma Aldrich, St Louis, MO, USA). Absorbance of cells was measured at $570 \mathrm{~nm}$ (BioTek ELx800 plate reader, Winooski, Vermont, USA).

\section{Proliferation assay}

Proliferation in MCF7 cells was determined by plating 6500 cells $/ \mathrm{cm}^{2}$ into 12 -well plate and cultured in a medium with and without ACV. After overnight incubation cells were detached with trypsin and counted using automated cell counter (TC20 ${ }^{\mathrm{TM}}, 1450102$, Bio-rad Laboratories, Berkeley, California) at 24, 48, 72 and $96 \mathrm{~h}$.

\section{Soft agar assay}

The ability of cancer cells to form colonies was characterized using a soft agar assay. This assay required 21 days of growth on the soft agar medium. At the end of 3 weeks, a number of colonies formed per petri dish were counted using a crystal violet stain. Briefly, 1\% sterile agar solution was warmed in a microwave and place to $37{ }^{\circ} \mathrm{C}$ water bath to cool down. $500 \mu \mathrm{g}$ of agarose 
powder (BP165-25, Thermo Fisher Scientific, Fair Lawn, New Jersey, USA) was dissolved in $50 \mathrm{~mL}$ distilled water. The bottom of the petri dish (502014-07P, Sterilin petri dishes $9.6 \mathrm{~cm}^{2}$, Dynalon Labware, Rochester, NY, USA) was coated with $0.7 \%$ agar and $0.3 \% \mathrm{CM}$ by adding $3 \mathrm{ml} /$ dish at room temperature for $30 \mathrm{~min}$. following this, the upper layer of $3 \mathrm{~mL}$ of agar solution with $0.3 \%$ agar and $0.7 \%$ cell suspension (3125 cells $/ \mathrm{cm}^{2}$ ) was plated. The top agar layer was allowed to solidify and then incubated for 3 weeks at $37{ }^{\circ} \mathrm{C}$ in $5 \% \mathrm{CO}_{2}$. $\mathrm{CM}$ was refreshed 2 times a week. In 21 days crystal violet was used as a staining for colonies (C3886, Sigma-Aldrich, Munich, Germany) and counting was performed using Leica DMI3000 B light microscope.

\section{Cell staining for fluorescent imaging Morphological analysis}

Glass coverslips were cleaned for $2 \mathrm{~h}$ in $200 \mathrm{~mL}$ ethanol, $50 \mathrm{~g} \mathrm{NaOH}$, and $300 \mathrm{~mL}$ DI water and finally rinsed with PBS. Cells were seeded on glass coverslips at 10,500 cells $/ \mathrm{cm}^{2}$, placed in petri dishes (Sterilin petri dishes $9.6 \mathrm{~cm}^{2}$ ) and incubated at $37{ }^{\circ} \mathrm{C}$ in $5 \% \mathrm{CO}_{2}$ overnight before the treatment. After the $72 \mathrm{~h}$ incubation with acyclovir, coverslips with cells were rinsed briefly with PBS for thrice for $5 \mathrm{~min}$ each time. 4\% paraformaldehyde was used as a fixative for $10 \mathrm{~min}$ at room temperature. The samples were blocked with 1\% BSA and $0.3 \%$ Tween-20 (P2287-500 ml, Sigma-Aldrich, St Louis, MO, USA) in PBS and incubated at room temperature for $1 \mathrm{~h}$. Following $1 \mathrm{~h}$ incubation, $\alpha$-tubulin rabbit mAb Alexa Fluor 488 conjugate (322588, Invitrogen, Life Technologies, Rockford, IL, USA) diluted as 1:200 was used for staining and incubation at $4{ }^{\circ} \mathrm{C}$ overnight in the dark. After $24 \mathrm{~h}$, the coverslips were washed 3 times for $5 \mathrm{~min}$ with PBS and then incubated with $0.1 \mu \mathrm{g} / \mathrm{mL}$ DAPI for $2 \mathrm{~min}$. After rinsing again with PBS, aqueous mounting medium (ab128982, Abcam, Cambridge, MA) was used for mounting coverslips on microscope slides. Finally, coverslips were sealed with a clear nail polish. Images were acquired using EVOS ${ }^{\bullet}$ FLoid ${ }^{\oplus}$ Cell Imaging Station (4471136, Life Technologies, Carlsbad, California, USA). Cellprofiler software was used to evaluate morphometric features of treated MCF7 cells (Broad Institute, www.cellprofiler.org). The equation used for the quantitative measurement of the shape of the cell is given below. This equation uses form factor, FF:

$$
\mathrm{FF}=4 * \pi * \text { area } / \text { perimeter }{ }^{2}
$$

The pipeline for this analysis included four modules: Identify Primary Objects, Identify Secondary Objects, Identify Tertiary Objects and Measure Object Size Shape [11].
Area ratio was calculated by dividing area of nucleus over area of cytoplasm.

Immunofluorescence staining for the presence of E-cadherin $4 \%$ paraformaldehyde in PBS was used as a fixative for $10 \mathrm{~min}$ at room temperature and $0.1 \%$ Triton was used as a permeabilization solution for $10 \mathrm{~min}$ on ice. Following this, cells were washed 3 times with ice cold PBS and blocked with 1\% BSA in PBST at room temperature for $1 \mathrm{~h}$. Blocked cells were stained with $1 \mathrm{mg} / \mathrm{ml}$ of $\mathrm{Ms}$ $\mathrm{mAb}$ to E-cadherin (ab1416, Abcam, Cambridge, MA, USA) in $1 \%$ BSA in PBST in a humidified chamber for $24 \mathrm{~h}$ at $4{ }^{\circ} \mathrm{C}$. The stained samples were then washed $3 \times 5$ min with PBS and incubated overnight with a $2 \mathrm{mg} / \mathrm{ml}$ of secondary antibody goat pAb to Ms IgG Alexa fluor 488 (ab150113, Abcam, Cambridge, MA, USA) in 1\% BSA in PBST. Coverslips were washed $3 \times 5 \mathrm{~min}$ with PBS in the dark and incubated with DAPI for $2 \mathrm{~min}$, and mounted on the microscope slides using glycerol. Images were acquired using EVOS $^{\oplus}$ FLoid $^{\oplus}$ Cell Imaging Station (4471136, Life Technologies, Carlsbad, California, USA).

\section{Aldehyde dehydrogenase activity colorimetric assay (ALDH)} NAD-dependent ALDH activity was measured using colorimetric assay kit (MAK082-1KT, Sigma Aldrich, Saint Louis, MO, USA) and performed as described in the manufacturer's instructions. The absorbance was measured at $450 \mathrm{~nm}$ on BioTek ELx800 plate reader. Measurements were recorded every $3 \mathrm{~min}$ until the value of the control sample exceeded the value of the most active standard (10 nmole/well). The following equation was used to calculate the activity of the enzyme:

$\underline{\text { ALDH Activity }=\text { NADH Amount (nmole) } \times \text { Sample Dilution Factor }}$

(Reaction time) $x$ Sample volume $(\mathrm{mL})$

\section{Transwell migration assay}

Cells were cultured for $72 \mathrm{~h}$ with and without acyclovir at $37{ }^{\circ} \mathrm{C}$ in $5 \% \mathrm{CO} 2$, and then in serum-free medium for another $24 \mathrm{~h}$ at $37{ }^{\circ} \mathrm{C}$ in $5 \% \mathrm{CO}_{2}$. After detachment with $0.05 \%$ trypsin- EDTA the cells were re-suspended in a serum-free medium. Upper insert was filled with $100 \mu \mathrm{l}$ of the cell suspension $\left(\sim 9 \times 10^{4}-1 \times 10^{6}\right.$ cells per well) while reservoir chamber was filled with $600 \mu$ l of culture medium. Migration of cells was monitored at 3, 6, and $12 \mathrm{~h}$ at $37{ }^{\circ} \mathrm{C}$ in $5 \% \mathrm{CO}_{2}$. Crystal violet was used as the staining solution to distinguish between migrated and non-migrated cells. A cotton swab was used to remove the cells that were left in the upper chamber of the membrane. Those cells that migrated through the insert were examined and counted with bright-field microscope (LEICA DMI3000 B, Wetzlar, Germany). 


\section{Wound healing}

Cell culture was performed at a concentration of $260,000 \mathrm{cells} / \mathrm{cm}^{2}$ in 12-well plates incubated overnight at $37{ }^{\circ} \mathrm{C}$ in $5 \% \mathrm{CO}_{2}$. The medium was then substituted with $\mathrm{CO}_{2}$ free media with and without ACV. A scratch using sharpened toothpick was made on the surface of the well to simulate a wound in vitro. Four randomly areas in a well were selected and imaged in 10 min intervals for $12 \mathrm{~h}$ using time-lapse microscopy system (AMAFD1000, EVOS FL Auto imaging system, Life Technologies, Thermo Fisher Scientific, Carlsbad, CA, USA). The images obtained were processed using ImageJ (image processing software). The sequence of images was analyzed and the open wound area was measured for each image at every hour. A scatter plot of wound area measurements (units) vs. time (in hours) was generated as seen in Additional data 5. A line-of-best fit was used to calculate the slope, which corresponded to the rate of migrated cells.

\section{E-cadherin, C-Myc, NF-kB p65 and caspase-3 levels colorimetric assays}

Secretion levels of E-cadherin in a cell culture were measured using a human E-cadherin ELISA colorimetric assay kit (99-1700, Invitrogen, Novex by Life Technologies, Frederick, MD, USA) and performed as described in the manufacturer's instructions. Cellular levels of CMyc, NF-kB p65 and caspase-3 in cell lysates were measured using a human C-Myc ELISA colorimetric assay kit (KHO 2041, Novex by Life Technologies, Frederick, MD, USA); a human NF-kB p65 Total ELISA colorimetric assay kit (KHO 0371, Novex by Life Technologies, Frederick, MD, USA) and a human caspase-3 ELISA Kit (KHO 1091, Novex by Life Technologies, Frederick, MD, USA).

Cell lysates were prepared using a $1 \mathrm{mM}$ phenylmethylsulfonyl fluoride (PMSF) cell extraction buffer, a protease inhibitor cocktail (78439, Thermo Scientific, Rockford, IL, USA) and RIPA buffer (89900, Thermo Scientific, Rockford, IL, USA). Lysis was performed by adding $500 \mu \mathrm{l}$ extraction buffer to the cell pellet for $30 \mathrm{~min}$ on ice while vortexing every $10 \mathrm{~min}$. Then the cells were placed in the microcentrifuge tubes at $13,000 \mathrm{rpm}$ for $10 \mathrm{~min}$ at $4{ }^{\circ} \mathrm{C}$. Lysates were stored at $-80{ }^{\circ} \mathrm{C}$. All experiments were performed in triplicate. Results were expressed as mean concentration \pm standard deviation and normalized to the number of live cells.

\section{Apoptosis assay}

Programmed cell death was studied using annexin V-FITC apoptosis detection kit (331200, Invitrogen, Camarillo, CA, USA). Treated and control cells were harvested and re-suspended in CM to obtain a target concentration of $1 \times 10^{6} / \mathrm{ml}$ in $1.5 \mathrm{ml}$ Eppendorf tubes.
Cells were centrifuged for $1 \mathrm{~min}$ at $3000 \mathrm{rpm}$, washed with ice cold PBS and centrifuged again. Cell pellets were re-suspended in $190 \mathrm{ul}$ of $1 \mathrm{x}$ binding buffer with $10 \mathrm{ul}$ Annexin V-FITC and $10 \mathrm{ul} \mathrm{of} 20 \mathrm{ug} / \mathrm{ml}$ propidium iodide for $15 \mathrm{~min}$ at room temperature in the dark. Apoptosis of the cells was analyzed by flow cytometry (SORP FACSAria -II with 6 lasers, BD Biosciences, San Jose, USA).

\section{Statistics}

All reported results below are presented as mean values \pm standard error values. To calculate differences between means, one-way analysis of variance (ANOVA) was implemented, where a null hypothesis was accepted when all means were equal. Population differences were calculated only for a treatment and a control within the cell line. If at least one mean was different, a follow-up Tukey's HSD test to compare between groups and calculate $\mathrm{p}$-values of each sample $(\alpha=0.05)$.

\section{Results}

Viability of MCF7 cells and breast epithelial cells after the treatment with $5 \mu \mathrm{M} \mathrm{ACV}$ were measured and normalized to the untreated culture cells [Additional file 1]. MCF7 cells and breast epithelial cells demonstrated 80,687 and $97,194 \%$ of viable cells after the ACV treatment, respectively.

First, we sought to evaluate the influence of ACV on the proliferation ability and apoptosis of MCF7 cells. Regulated interplay between apoptosis and cell proliferation is essential for the processes like tissue development and deregulation mechanisms [12]. Deregulated mechanism of apoptosis that correlates with an uncontrolled cell proliferation eventually leads to the carcinogenesis [13]. Several antiviral agents demonstrated the ability to inhibit proliferation and increase proapoptotic activity of the cancer cells [2]. Here, the rate of proliferation decreased during $96 \mathrm{~h}$ treatment with ACV (Fig. 1a). ACV significantly increased a population doubling time compared to the control cells $\bowtie 1.4$ fold [Additional file 2]. Caspase-3 level in MCF7 cells was estimated to evaluate the apoptotic activity of cancer cells in response to ACV treatment. ACV upregulated caspase- 3 expression in the treated cells $\sim 1.7$ compared to the control cells $(p<0.05)$ (Fig. 1b). Annexin $\mathrm{V}$ staining and flow cytometry analysis demonstrated a slight increase of the number of the apoptotic cells in response to ACV treatment ( $p>0.05$ for the late and early apoptosis) (Fig. 1c, Additional file 3).

When examining normal cells and cancerous cells under the microscope, we observed distinctive external characteristic features. Results of the IF staining indicate that cancer cells underwent changes in their morphological characteristics in response to ACV treatment (Fig. 1d). FF shape descriptor was used quantitative 


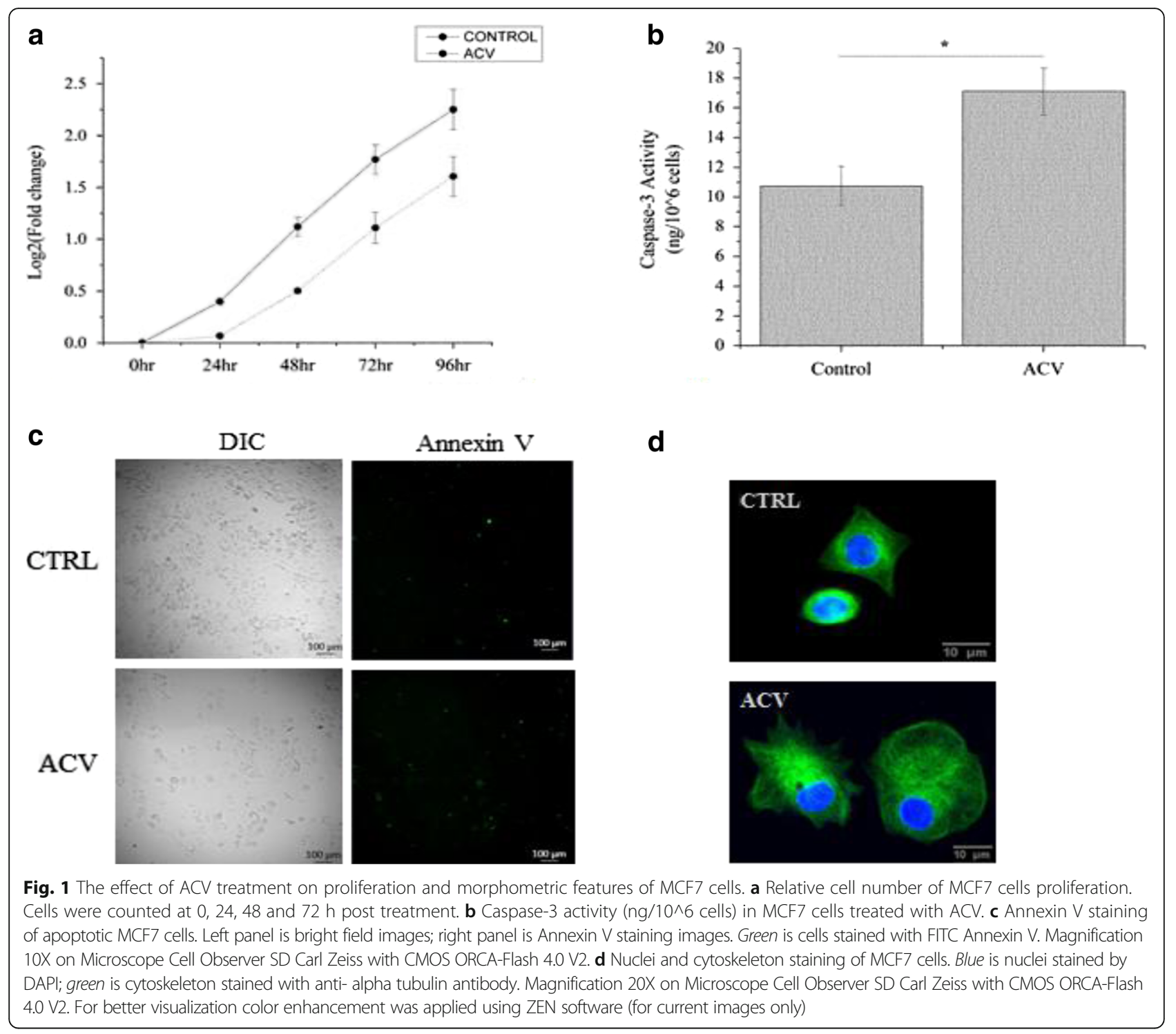

characterization of these changes, where FF value of 1 served as a detector of a circular shape and 0 indicated linear or star shaped object [Additional file 4]. ACV treated cancer cells displayed a decrease of FF compared to the control cells from $0.828 \pm 0.014$ to $0.659 \pm 0.012$, indicating that ACV treated cells were more spread out with a non-uniform shape ( 01.25 fold). Furthermore, ACV treated cancer cells had a larger cytoplasmic volume compared to the control cells.

The effect of ACV treatment on the migratory and invasive capacities of the breast cancer cells was also tested. Various environmental factors can modulate the motility of cancer cells and affect invasion capacity of these cells. Teng et al. showed that antiviral drug ribavirin causes a considerable suppression of the migration of renal cell carcinoma cell lines [14]. Boyden chamber migration assay was performed to assess whether ACV affects MCF7 chemopolarised migration. As seen in Fig. 2a, ACV treatment reduces the number of cells migrating towards the chemoattractants as compared to the control cells. The cell invasion capacity of the treated cancer cells dropped $\sim 15$ times compared to the untreated cells $(p<0.05)$. Fig. $2 \mathrm{~b}$ shows the effect of $\mathrm{ACV}$ on the collective motility and rates of migration of both normal and cancer cells. The rate of the wound closure of ACV treated cancer cells decreased significantly compared to the control cells with $\sim 1.34$ fold and was comparable to the rate of normal breast epithelial cells $(24.74 \mu \mathrm{m} / \mathrm{h}$ and $21.95 \mu \mathrm{m} / \mathrm{h}$ for ACV treated and normal cells, respectively) $(p<0.05)$ [Additional file 5].

E-cadherin is secreted in most of the epithelial tissues and normal expression of E-cadherin has been reported to inhibit metastasis and invasion by suppressing epithelialmesenchymal transition as well as stimulating cell-cell 


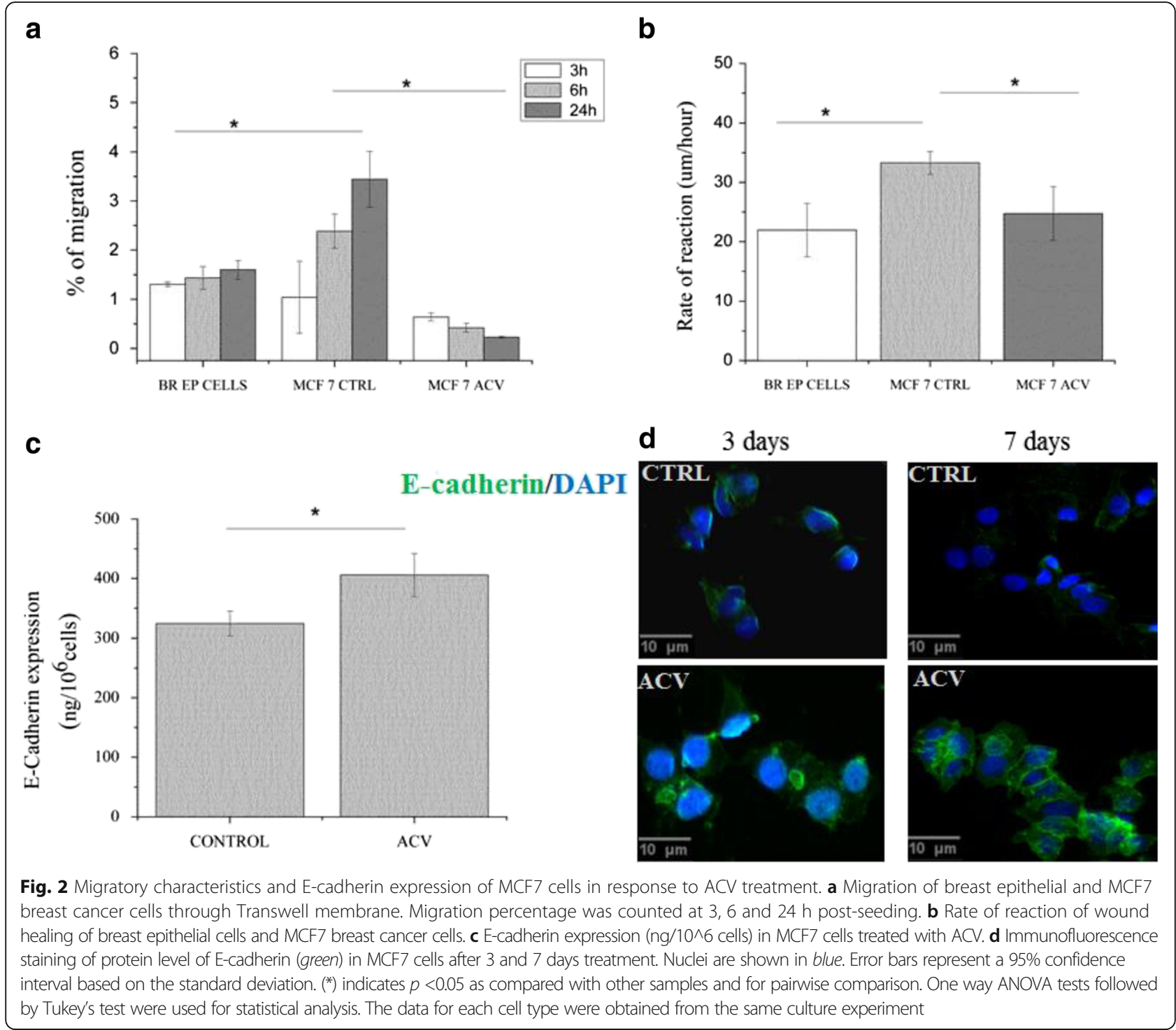

adhesions $[15,16]$. We observed a significant increase of E-cadherin secretion in ACV treated cells compared to the control $\sim 1,25(p<0.05)$ as shown in Fig. 2c, d.

Next, we evaluated the action of ACV on the ability of MCF7 cells to form colonies. This distinguishing feature of the cell transformation and its deregulated growth served as a marker to distinguish between cancer and normal cells, since normal cells do not have the ability to grow in semisolid matrices [17]. ACV treatment effectively decreased ( $\infty 2$ fold) the number of colonies formed by cancer cells compared to the untreated cells as seen in Fig. 3a. Also the differences in external features are clearly observable in rough scabrous surface of control cells ( $R$ - shape) versus smoother surface of ACV treated cells (S -shape). (Fig. 3b).

The concentration changes of C-Myc protein secreted by MCF7 cells were determined in response to $\mathrm{ACV}$ treatment. C-Myc oncogene regulates cellular growth and metabolic mechanisms as well as their interconnection [18]. Our results demonstrate a non-significant effect of ACV on the level of secretion of C-Myc in MCF7 cells. The concentration of C-Myc protein was similar to the control (Fig. 4a). Additional examinations of the viral protein NF-kB p65 demonstrated an elevated level after treatment with ACV [Additional file 6].

ALDH activity is one of the detectors of cancer progression [19]. Upregulated expression of ALDH1, one of the isoforms of ALDH family, has been reported as a crucial event in the breast cancer prognosis correlated with a poor clinical outcome [20,21]. Moreover, studies show that ALDH activity is linked to the differentiation and expansion. It is also associated with a self-protective ability [22]. Our assessment of the effect of ACV treatment on ALDH activity of breast cancer cells shows a 


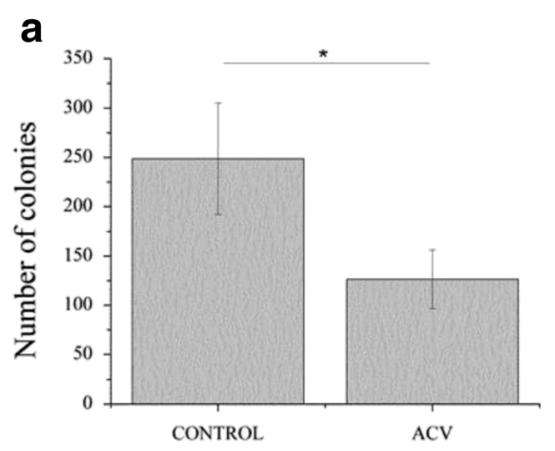

b

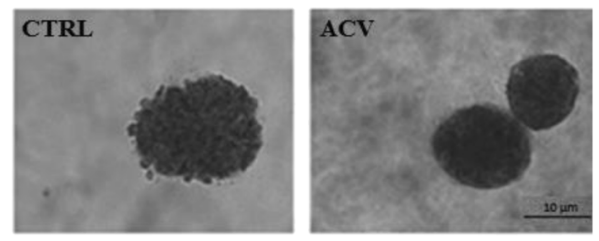

Fig. 3 ACV altered ability to form colonies of MCF7 breast cancer cells (a) Number of colonies of MCF7 cells in response to ACV treatment. b Representative images of colonies formed. Duration of growing on a soft agar was 21 days. Error bars represent a 95\% confidence interval based on the standard deviation. (*) indicates $p<0.05$ compared with control and other samples. One way ANOVA followed by Tukey's test were used for statistical analysis. The data for each cell type were taken from the same culture experiment

significant decrease ( 3 fold) of ALDH activity in MCF7 cells compared to the control cells (Fig. 4b).

\section{Discussion}

Recently, several antiviral agents have been found to possess the ability to decrease the rate of the cells' proliferation and to promote apoptosis in cancer cells. However, despite the compelling results supporting the clinical use of antiviral agents as an adjuvant therapy in cancer treatment, there is still a lack of studies of the biochemical mechanisms of their anticancer effects [23, 24]. There are several factors that might be involved in a therapeutic approach of the antiviral adjuvant therapy in cancer treatment: an antiviral agent selectively targets cancer related viruses or post-chemotherapy infections and may also result in the cytotoxic and antiproliferative effects on the cancer cells, causing apoptosis [2]. Our implementation of $\mathrm{ACV}$ as an antiviral strategy demonstrated a positive effect on the prevention as well as successful predictive capability in the treatment of various types of malignancies.
In this study, we used acyclovir (ACV) to examine the potential of the antiviral treatment on MCF7 breast cancer cell line. Acyclovir is an antiviral drug used in treating infections of Herpesviridae family [25]. In several studies antiviral agents similar to ACV were used as an adjuvant therapy along with the chemotherapy [26, 27]. Records of the patients diagnosed with nasopharyngeal carcinoma demonstrate a suppression of the tumor growth for several months where injection of antiviral was used in tandem with the chemotherapy [26]. Moreover, in situ hybridization shows that the tumor cell populations were reduced in EBV-encoded RNAs [26]. In another study, the effect of acyclic nucleoside phosphonate against HPV-associated cancer was examined. The results indicate that the adjunct therapy using a cytotoxic drug and acyclic nucleoside phosphonates is more effective than one therapy alone. The authors also report an inhibited rate of the virus replication that led to a decreased expression of the viral oncoproteins and upregulation of the tumor-suppressor genes [27].

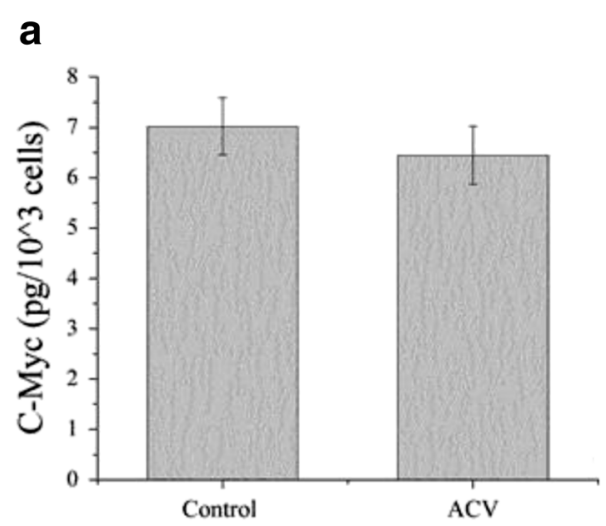

b

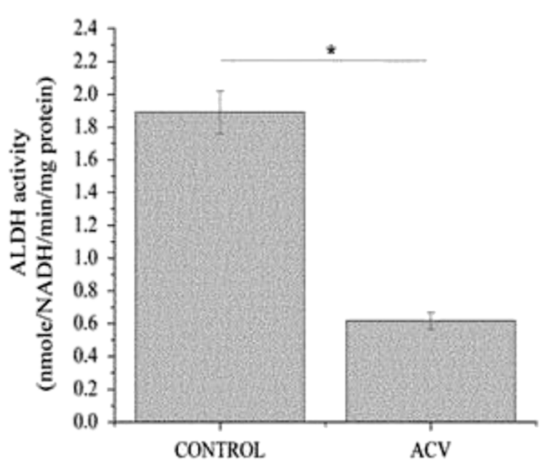

Fig. 4 a C-Myc (pg/10^3 cells) and (b) ALDH activity (nmol NADH/min/mg protein) expressions of MCF7 cells in response to ACV treatment. Error bars represent $95 \%$ confidence interval based on the standard deviation. $\left(^{*}\right)$ indicate $p<0.05$ as compared with other samples and for pairwise comparison. One way ANOVA tests followed by Tukey's test were used for statistical analysis. The data for each cell type were obtained from the same culture experiment 
Based on our results, we conclude that $\mathrm{ACV}$ as an antiviral agent has a potential suppressive effect on MCF7 breast cancer cells. ACV does not affect viability of non-cancerous breast epithelial cells, while showing a decrease of the viability of MCF7 breast cancer cells. Observed morphological changes and apoptosis analysis demonstrated the ability of ACV to affect the process of programmed cell death of MCF7 cells. The mechanism of apoptosis requires a number of proteins that regulate a proper cell death. One of these proteins is caspase-3 which is included in a family of cysteine proteases [28]. An upregulated level of the apoptosis associated cytokine Caspase- 3 was detected in ACV treated cells, correlating with the higher number of apoptotic cells and decreased rate of the cancer cell proliferation. Previously, it was reported that zidovudine treatment combined with a chemotherapeutic agent cisplatin has increased the apoptosis level of head and neck cancer cells [29]. This synergistic strategy of zidovudine and cisplatin was shown to trigger abnormal regulation of the mitochondria, increase of oxidative stress response and cause a significant cytotoxic effect on the cancer cells through the inhibition of a thiol metabolism [29]. Quantitative analysis revealed a moderate effect of acyclovir with a slight increase of the apoptotic cells.

$\mathrm{ACV}$ was also able to decrease the rate of the growth, colony formation ability, and cell invasion capacity of MCF7 breast cancer cells. These observations correlate with an upregulated secretion of E-cadherin in ACV treated cells. As previously mentioned, E-cadherin is an essential marker in the building of cell-to-cell adhesion and downregulation of this protein leads to the stimulation of invasion and metastasis [30].

Previous studies also report that antiviral agents can affect the secretion of the specific translation initiation factors, oncogenes, and angiogenic genes [8, 31, 32]. Borden et al. showed the ability of ribavirin to decrease the oncogenic potential of eukaryotic translation initiation factor (eIF4E) in the case of acute myeloid leukemia with a poor prognosis. Ribavirin binds eIF4E around the $\mathrm{m} 7 \mathrm{G}$ cap-binding site leading to the reduction of affinity of this translation initiator factor [32]. While, we observed that ACV did not affect the expression levels of C-Myc oncogene, suggesting that antiviral agents might have a selective impact on the secretion of specific proteins.

Moreover, our study showed that acyclovir was able to influence ALDH activity in the breast cancer cells. The ALDH superfamily consists of 19 isoenzymes with various cellular localizations, tissue/organ distributions and functions. ALDH enzymes catalyze highly reactive aldehydes and some isoenzymes play structural roles related to the osmoregulation and possess antioxidant functions [30]. Following cancer stem cell theories, where cancer is suggested to have a stem origin, ALDH was found to be a common marker for both normal and cancer stem cells [22, 33, 34]. Increased level of ALDH enzyme is an indicator of a high tumorigenic potential of the cancer cell and ability to self-renew and initiate tumor progression [21]. In the breast cancer cells upregulated expression of ALDH is associated with a poor clinical outcome $[19,20]$. Our results suggest that ACV affects multiple aspects of cellular life related to the carcinogenesis and ALDH fulfills the role of a marker for these changes.

A study by Curiel et al. reported that ACV had an inhibitory effect on one of the immune system components as T-regulatory cells (Treg) in glioblastomas through the suppression of indoleamine 2, 3-dioxygenase activity [35]. Another antiviral agent - ribavirin was also reported as an immune response inducer in the renal cell carcinoma lines through the downregulation of IL-10 expression and the upregulation of TGF- $\beta$ expression [14]. The mechanisms by which ACV enables its anticancer effects might involve an enhanced immune response of the cancer cells and further study is required in this area.

There are several limitations in this study. All experiments were performed in vitro only on one cell line. Future research should focus on an adjuvant strategy of different antiviral agents to determine whether a combinatorial effect exists, and if so, which pathways are affected during the mechanism. Additionally, an examination of epigenetic modifications might serve as a platform for understanding the molecular mechanism underlying the antiviral therapy.

\section{Conclusion}

In summary, we present evidence that $\mathrm{ACV}$ has an anticancer effect on breast cancer cell line. Our study shows that ACV was able to inhibit cancer cells proliferation, colony formation ability and cell invasion capacity, while having no effect on the secretion of certain tumor suppressor genes. Treatment with ACV induced downregulation of ALDH activity, suggesting a decrease of the tumorigenic potential of the treated cancer cells. These results provide new insights on the effect of antiviral agents on the tumorigenesis and metastasis. However, more research is necessary to identify the primary target of ACV and maximize its potential.

\section{Additional files}

Additional file 1: Viability of MCF7 breast cancer and normal breast epithelial cells in response to acyclovir. Error bars represent $95 \%$ confidence interval based on the standard deviation. $\left(^{*}\right)$ indicates $p<0.05$ as compared with other samples and for pairwise comparison. One way ANOVA followed by Tukey's test were used for statistical analysis. The data for each cell type were taken from same culture experiment. (DOCX $144 \mathrm{~kb}$ )

Additional file 2: Population doubling time (hours) of proliferation of MCF7 cells treated with ACV. Error bars represent 95\% confidence interval 
based on the standard deviation. $\left.{ }^{*}\right)$ indicates $p<0.05$ as compared with other samples and for pairwise comparison. One way ANOVA followed by Tukey's test were used for statistical analysis. The data for each cell type were taken from same culture experiment. (DOCX $301 \mathrm{~kb}$ )

Additional file 3: Annexin V staining of apoptotic MCF7 cells after treatment with acyclovir. Left panel is early apoptosis, right panel is late apoptosis. Error bars represent 95\% confidence interval based on the standard deviation. One way ANOVA followed by Tukey's test were used for statistical analysis. Means are not significant, $p>0.05$. $P$-value for early apoptosis $=1.31579$; for late apoptosis $=0.91371$. The data for each cell type were taken from same culture experiment. (DOCX $288 \mathrm{~kb}$ )

Additional file 4: Quantitative analysis of nucleus and cytoplasm of MCF7 breast cancer cells without and with acyclovir treatment. (DOCX $10 \mathrm{~kb}$ )

Additional file 5: A scatter plot of measurements where best fit line and a slope indicate rate of migrating cells. (DOCX $62 \mathrm{~kb}$ )

Additional file 6: NF-kB p65 (pg/10^3 cells) expression of MCF7 cells in response to ACV treatment. Error bars represent $95 \%$ confidence interval based on the standard deviation. $\left(^{*}\right)$ indicates $p<0.05$ as compared with other samples and for pairwise comparison. One way ANOVA followed by Tukey's test were used for statistical analysis. The data for each cell type were taken from the same culture experiment. (DOCX $28 \mathrm{~kb}$ )

\section{Abbreviations}

ACV: Acyclovir; ALDH: aldehyde dehydrogenase; BCS: Bovine calf serum BSA: Bovine serum albumin; CMV: Cytomegalovirus; DMEM: Dulbecco's modified eagle's medium; DMSO: Dimethyl sulfoxide; EBV: Epstein-barr virus; elF4E: Eukaryotic translation initiation factor 4E; ELISA: Enzyme-linked immunosorbent assay; EMT: Epithelial-to-mesenchymal transition; FF: Form factor; HBV: Hepatitis B; HCC: Hepatocellular carcinoma; HCV: Hepatitis C; hENT1: Human equilibrative nucleoside transporter 1; HPV: Human papillomavirus; HSV: Herpes simplex virus; IF: Immunofluorescence; MTT: 3-(4,5-dimethylthiazol-2yl)-2,5-diphenyltetrazolium bromide; NAD: Nicotinamide adenine dinucleotide; NF-kB: Nuclear factor kappa-light-chain-enhancer of activated B cells; PBS: Phosphate buffered saline; PMSF: Phenylmethylsulfonyl fluoride; RIPA buffer: Radioimmunoprecipitation assay buffer; Treg: T-regulatory cells

\section{Acknowledgment}

Authors would like to thank Samal Zhussupbekova, Sabina Murzakhmetova and Tomiris Atazhanova for their help with the collection of colony formation, cell migration and IF staining data.

\section{Funding}

This work was supported by №0121-1 Program-oriented funding-13 by the Ministry of Education and Science of the Republic of Kazakhstan.

\section{Availability of data and materials}

The datasets used and/or analyzed during the current study available from the corresponding author on reasonable request.

\section{Authors' contributions}

MS, KA, and DA. designed the study. MS, OK and DM performed experiments. MS, OK, KA and DA. analyzed data. MS and DA wrote the manuscript. All authors read and approved the final manuscript.

\section{Competing interests}

Authors declare that they have no competing interests.

\section{Consent for publication}

Not applicable.

\section{Ethics approval and consent to participate}

Not applicable.

\section{Publisher's Note}

Springer Nature remains neutral with regard to jurisdictional claims in published maps and institutional affiliations.

\section{Author details}

National Laboratory Astana, Nazarbayev University, Qabanbay Batyr Avenue 53, Astana 010000, Kazakhstan. ${ }^{2}$ Nazarbayev University Research and Innovation System, Nazarbayev University, Astana, Kazakhstan. ${ }^{3}$ Locus Solutions LLC, Solon, OH, USA

Received: 5 December 2016 Accepted: 16 March 2017

Published online: 23 March 2017

\section{References}

1. Shih WL, Fang CT, Chen PJ. Anti-viral treatment and cancer control. Recent Results Cancer Res. 2014;193:269-90.

2. Alibek K, Bekmurzayeva A, Mussabekova A, Sultankulov B. Using antimicrobial adjuvant therapy in cancer treatment: a review. Infect Agent Cancer. 2012;7(1):33.

3. Alibek K, Kakpenova A, Baiken Y. Role of infectious agents in the carcinogenesis of brain and head and neck cancers. Infect Agent Cancer. 2013;8(1):7.

4. Cathomas G. Kaposi's sarcoma-associated herpesvirus (KSHV)/human herpesvirus 8 (HHV-8) as a tumour virus. Herpes. 2003;10(3):72-7.

5. Gordon SC, et al. Antiviral therapy for chronic hepatitis B virus infection and development of hepatocellular carcinoma in a US population. Clin Gastroenterol Hepatol. 2013;12(5):885-93.

6. Pettersson F, et al. Ribavirin treatment effects on breast cancers overexpressing elF4E, a biomarker with prognostic specificity for luminal B-type breast cancer. Clin Cancer Res. 2011:17(9):2874-84.

7. Assouline $\mathrm{S}$, et al. Molecular targeting of the oncogene elF4E in acute myeloid leukemia (AML): A proof-of-principle clinical trial with ribavirin. Blood. 2009;114(2):257-60.

8. Kentsis A, Topisirovic I, Culjkovic B, Shao L, Borden KLB. Ribavirin suppresses elF4E-mediated oncogenic transformation by physical mimicry of the 7-methy guanosine mRNA cap. Proc Natl Acad Sci U S A. 2004;101(52):18105-10.

9. Namba T, Kodama R, Moritomo S, Hoshino T, Mizushima T. Zidovudine, an anti-viral drug, resensitizes gemcitabine-resistant pancreatic cancer cells to gemcitabine by inhibition of the Akt-GSK3ß-Snail pathway. Cell Death Dis. 2015;6:e1795.

10. Elion GB. Mechanism of action and selectivity of acyclovir. Am J Med. 1982; 73(1 PART 1):7-13.

11. Bygd HC, Akilbekova D, Muñoz A, Forsmark KD, Bratlie KM. Poly-l-arginine based materials as instructive substrates for fibroblast synthesis of collagen. Biomaterials. 2015:63:47-57.

12. Alenzi FQB. Links between apoptosis, proliferation and the cell cycle. Br J Biomed Sci. 2004;61(2):99-102.

13. Evan Gl, Vousden KH. Proliferation, cell cycle and apoptosis in cancer. Nature. 2001;411(6835):342-8.

14. Teng $L$, et al. Anti-tumor effect of ribavirin in combination with interferon-a on renal cell carcinoma cell lines in vitro. Cancer Cell Int. 2014;14:63.

15. Singhai R, Patil WW, Jaiswal SR, Patil SD, Tayade MB, Patil AV. E-Cadherin as a diagnostic biomarker in breast cancer. N Am J Med Sci. 2011;3(5):227-33.

16. Kowalski PJ, Rubin $\mathrm{M}$ a, Kleer CG. E-cadherin expression in primary carcinomas of the breast and its distant metastases. Breast Cancer Res. 2003;5(6):R217-22.

17. Borowicz S, et al. The soft agar colony formation assay. J Vis Exp. 2014;92: e51998.

18. Miller DM, Thomas SD, Islam A, Muench D, Sedoris K. c-Myc and cancer metabolism. Clin Cancer Res. 2012;18(20):5546-53.

19. Marcato P, Dean CA, Giacomantonio CA, Lee PWK. Aldehyde dehydrogenase its role as a cancer stem cell marker comes down to the specific isoform. Cell Cycle. 2011;10(9):1378-84

20. Chaterjee M, van Golen KL. Breast cancer stem cells survive periods of farnesyl-transferase inhibitor-induced dormancy by undergoing autophagy. Bone Marrow Res. 2011;2011:362938.

21. Ginestier $\mathrm{C}$, et al. ALDH1 is a marker of normal and malignant human mammary stem cells and a predictor of poor clinical outcome. Cell Stem Cell. 2007;1(5):555-67.

22. Ma I, Allan AL. The role of human aldehyde dehydrogenase in normal and cancer stem cells. Stem Cell Rev. 2011;7(2):292-306.

23. Söderlund J, Erhardt S, Kast RE. Acyclovir inhibition of IDO to decrease Tregs as a glioblastoma treatment adjunct. J Neuroinflammation. 2010;7:44.

24. Zhou FX, et al. Radiosensitization effect of zidovudine on human malignant glioma cells. Biochem Biophys Res Commun. 2007;354(2):351-6.

25. Spruance SL, Nett R, Marbury T, Wolff R, Johnson J, Spaulding T. Acyclovir cream for treatment of herpes simplex labialis: Results of two randomized 
double-blind, vehicle-controlled, multicenter clinical trials. Antimicrob Agents Chemother. 2002;46(7):2238-43.

26. Yoshizaki T, et al. Treatment of locally recurrent Epstein-Barr virus-associated nasopharyngeal carcinoma using the anti-viral agent cidofovir. J Med Virol. 2008;80(5):879-82.

27. Abdulkarim B, Bourhis J. Antiviral approaches for cancers related to EpsteinBarr virus and human papillomavirus. Lancet Oncol. 2001;2(10):622-30.

28. Devarajan $\mathrm{E}$, et al. Down-regulation of caspase 3 in breast cancer: a possible mechanism for chemoresistance. Oncogene. 2002;21(57):8843-51.

29. Mattson DM, et al. Cisplatin combined with zidovudine enhances cytotoxicity and oxidative stress in human head and neck cancer cells via a thiol-dependent mechanism. Free Radic Biol Med. 2009;46(2):232-7.

30. Hanahan D, Weinberg RA. Hallmarks of cancer: the next generation. Cell. 2011;144(5):646-74.

31. Flore O, Rafii S, Ely S, O'Leary JJ, Hyjek EM, Cesarman E. Transformation of primary human endothelial cells by Kaposi's sarcoma-associated herpesvirus. Nature. 1998;394(6693):588-92.

32. Borden KLB, Culjkovic-Kraljacic B. Ribavirin as an anti-cancer therapy: acute myeloid leukemia and beyond? Leuk Lymphoma. 2010;51(10):1805-15

33. Sell S. Stem cell origin of cancer and differentiation therapy. Crit Rev Oncol Hematol. 2004;51(1):1-28.

34. Reya T, Morrison SJ, Clarke MF, Weissman IL. Stem cells, cancer, and cancer stem cells. Nature. 2001;414(6859):105-11.

35. Curiel TJ. Tregs and rethinking cancer immunotherapy. J Clin Investig. 2007; 117(5):1167-74.

\section{Submit your next manuscript to BioMed Central and we will help you at every step:}

- We accept pre-submission inquiries

- Our selector tool helps you to find the most relevant journal

- We provide round the clock customer support

- Convenient online submission

- Thorough peer review

- Inclusion in PubMed and all major indexing services

- Maximum visibility for your research

Submit your manuscript at www.biomedcentral.com/submit 